\title{
Transmission Electron Microscopy Specimen Preparation for Two Dimensional Material Using Electron Beam Induced Deposition of a Protective Layer in the Focused Ion Beam Method
}

\author{
Byeong-Seon An, Yeon Ju Shin ${ }^{1}$, Jae-Seon Ju${ }^{1}$, Cheol-Woong Yang* \\ School of Advanced Materials Science \& Engineering, Sungkyunkwan University, Suwon 16419, Korea \\ ${ }^{1}$ Cooperative Center for Research Facilities, Sungkyunkwan University, Suwon 16419, Korea
}

*Correspondence to: Yang CW,

(iD) http://orcid.org/0000-0003-0475-8399

Tel: $+82-31-290-7362$

Fax: +82-31-290-7371

E-mail: cwyang@skku.edu

Received December 3, 2018

Revised December 21, 2018

Accepted December 21, 2018

\begin{abstract}
The focused ion beam (FIB) method is widely used to prepare specimens for observation by transmission electron microscopy (TEM), which offers a wide variety of imaging and analytical techniques. TEM has played a significant role in material investigation. However, the FIB method induces amorphization due to bombardment with the high-energy gallium $\left(\mathrm{Ga}^{+}\right)$ion beam. To solve this problem, electron beam induced deposition (EBID) is used to form a protective layer to prevent damage to the specimen surface. In this study, we introduce an optimized TEM specimen preparation procedure by comparing the EBID of carbon and tungsten as protective layers in FIB. The selection of appropriate EBID conditions for preparing specimens for TEM analysis is described in detail.
\end{abstract}

Key Words: 2-D materials, Focused ion beam (FIB), Electron beam induced deposition (EBID)

\section{INTRODUCTION}

Two-dimensional (2-D) materials, including graphene, $\mathrm{MoS}_{2}$, $\mathrm{WS}_{2}$, and h-BN, are crystalline films consisting of a single layer of atoms. Since these materials exhibit a variety of interesting properties from high-band gap insulators to semiconductors and semi-metals, they are widely used in electronic and photonic applications in batteries and electrochemical solar cells. The microstructural, chemical, and electrical properties of 2-D materials are typically characterized by analytical tools such as Raman spectroscopy, X-ray photoelectron spectroscopy (XPS), atomic force microscopy (AFM), and transmission electron microscopy (TEM). Among these methods, TEM equipped with additional analytical attachments is a powerful technique to obtain accurate information regarding the microstructure, interfaces, thickness, and chemical composition of the material at the nanoscale. Because of these useful functions, TEM analysis has been widely used in the fields of ma- terials science and engineering. However, to obtain accurate data using TEM analysis, the specimen should be made very thin so the electron beam can easily penetrate the specimen. In general, ion milling (John \& Robert, 1984), mechanical flat and wedge polishing (Cha et al., 2016; Mkhoyan et al., 2006; Okuno et al., 2008), and focused ion beam (FIB) (Giannuzzi \& Stevie, 1999; Stevie et al., 1995) are used as methods for preparing TEM specimens (Williams \& Carter, 2009).

The ion milling method, a commonly used preparation method for TEM specimens, can be used to strip off the surface layer by firing argon $\left(\mathrm{Ar}^{+}\right)$or xenon $\left(\mathrm{Xe}^{+}\right)$ions at a particular angle after mechanical polishing. However, it is difficult to prepare patterned samples such as actual electronic devices and materials with poor adhesion (e.g. interface between a 2-D material and $\mathrm{SiO}_{2}$ ).

On the other hand, FIB has been extensively used in the semiconductor industry and field of materials science because the specimen preparation is facile without any of the restrictions

(a) This is an open-access article distributed under the terms of the Creative Commons Attribution Non-Commercial License (http://creativecommons.org/licenses/by-nc/4.0) which permits unrestricted noncommercial use, distribution, and reproduction in any medium, provided the original work is properly cited.

Copyrights @ 2018 by Korean Society of Microscopy 
mentioned above. However, the FIB method forms a damaged layer as a result of bombardment by highly accelerated gallium $\left(\mathrm{Ga}^{+}\right)$ions. This damage typically appears in the form of amorphization on the surface on the TEM specimen. To solve this problem, low-energy $\mathrm{Ga}^{+}$or $\mathrm{Ar}^{+}$ion milling as final step is used to minimize the damage caused by the high-energy $\mathrm{Ga}^{+}$ion beam. In addition, electron beam induced deposition (EBID) can be used to gently deposit a protective layer on the surface before forming the lamellar structure (Kato, 2004). Carbon, platinum, and tungsten materials are generally used as a depositing source in EBID. The representative platinum-EBID protective layer is known to prevent damage caused by collisions between $\mathrm{Ga}^{+}$ions and the surface but it is very expensive. Alternatively, carbon and tungsten-EBID can be used as inexpensive protective layers. However, studies on inexpensive $\mathrm{C}$ and $\mathrm{W}$-based protective layers have not been reported to date.

Therefore, in this study, we introduce an optimized TEM specimen preparation procedure using FIB by comparing carbon and tungsten as a depositing source in EBID for the generation of inexpensive protective layers.

\section{MATERIALS AND METHODS}

A thin molybdenum film was deposited on a transferred CVD-grown graphene substrate by e-beam evaporation. $\mathrm{MoS}_{2}$ was synthesized by sulfurizing at $300^{\circ} \mathrm{C}$ under a $\mathrm{H}_{2} \mathrm{~S}$ and Ar plasma atmosphere for $1.5 \mathrm{~h}$ in the plasma-enhanced chemical vapor deposition(PECVD) (Ahn et al., 2015).

The crystallinity of the PECVD-grown $\mathrm{MoS}_{2}$ was characterized by Raman spectroscopy at $532 \mathrm{~nm}$ (Alpha300 M+; WITec Gmbh Inc., Germany). The plan-view TEM specimens were prepared by rinsing with deionized water (D.I. water) and floating the $\mathrm{MoS}_{2}$ on $\mathrm{SiO}_{2}$ substrate on a diluted hydrofluoric acid (HF) solution using a conventional transfer method to characterize the crystallinity of the PECVD-grown $\mathrm{MoS}_{2}$. The $\mathrm{SiO}_{2}$ layer was etched using a diluted HF solution, detaching the $\mathrm{MoS}_{2}$ from the underlying $\mathrm{SiO}_{2}$ substrate. Finally, the detached $\mathrm{MoS}_{2}$ films were transferred to a TEM grid.

To compare TEM samples prepared with carbon- and tungsten-EBID as protective layers to prevent the bombardment by $\mathrm{Ga}^{+}$ions during FIB, cross-sectional TEM specimens were fabricated via FIB (NX2000; Hitachi Inc., Japan) using the

A

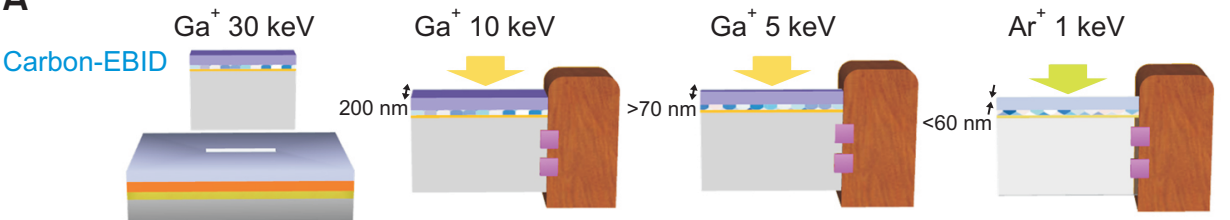

B

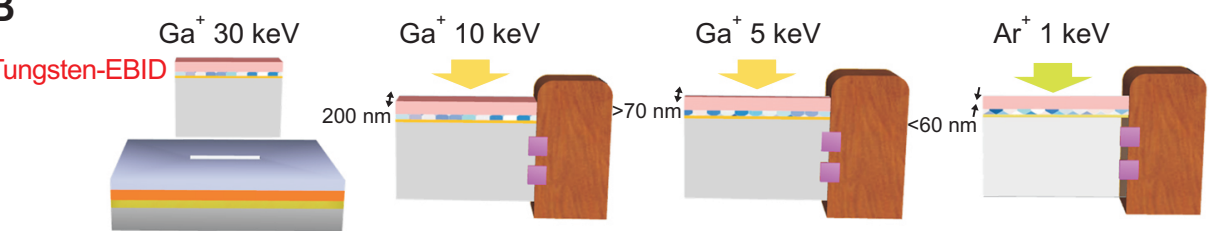

Fig. 1. Schematics for preparation of TEM specimen fabricated using (A) carbonEBID and (B) tungsten-EBID as protective layer in FIB method.
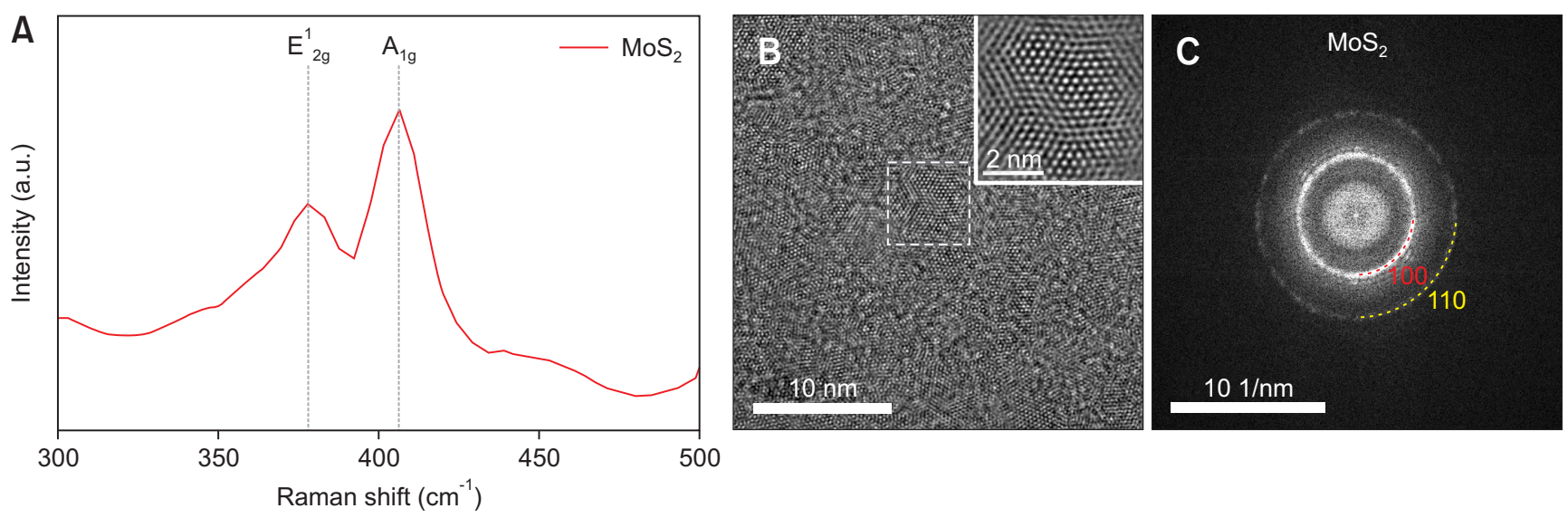

Fig. 2. (A) Representative Raman spectra of $\mathrm{MoS}_{2}$ (B) the plan-view HR-TEM micrograph of $\mathrm{MoS}_{2}$ (C) FFT corresponding to plan-view HR TEM micrograph. 
lift-out technique, as shown in Fig. 1. Carbon- and tungstenEBID were deposited using the focused electron beam and gas injection system (GIS) to form a protective layer. The TEM samples were etched using a high-energy $\mathrm{Ga}^{+}$ion beam at 30 $\mathrm{keV}$ and $1.5 \mathrm{nA}$ until reaching thickness of $\sim 500 \mathrm{~nm}$ and were subsequently thinned to a foil thickness of $\sim 70 \mathrm{~nm}$ at $5 \sim 10$ $\mathrm{keV}$ and $40 \mathrm{pA}$. As the final step, a low-energy $\mathrm{Ar}^{+}$ion beam at $1 \mathrm{keV}$ and $19 \mathrm{nA}$ was used to minimize the damage to the surface layers.

The prepared samples were investigated by analytical TEM (JEM-ARM200F; JEOL, Japan) at $80 \mathrm{keV}$ to avoid electron beam-induced damage to the samples during TEM observation (Garcia et al., 2014). High-resolution (HR) images were recorded using a CCD camera (Oneview; Gatan Inc., USA).

\section{RESULTS AND DISCUSSION}

Fig. 2 shows the Raman spectra acquired from $\mathrm{MoS}_{2}$ excited at $532 \mathrm{~nm}$, plan-view HRTEM images, and fast Fourier transform (FFT). As shown in Fig. 2A, the in-plane $\left(\mathrm{E}_{1 \mathrm{~g}}^{1}, 2383\right.$ $\mathrm{cm}^{-1}$ for bulk $\left.\mathrm{MoS}_{2}\right)$ and out-of-plane vibrations $\left(\mathrm{A}_{1 \mathrm{~g}}, \sim 408\right.$ $\mathrm{cm}^{-1}$ for bulk $\mathrm{MoS}_{2}$ ) modes in the PECVD-grown $\mathrm{MoS}_{2}$ were observed at 378 and $406 \mathrm{~cm}^{-1}$, respectively. Fig. $2 \mathrm{~B}$ and $\mathrm{C}$ shows the plan-view HRTEM image and the corresponding FFT. In the plan-view HRTEM image, the lattice arrangement of the PECVD-grown $\mathrm{MoS}_{2}$ adopted a hexagonal structure nanocrystalline grains $\sim 5 \mathrm{~nm}$ in size were observed (inset of Fig. 2B). These results confirmed that $\mathrm{MoS}_{2}$ was fully synthesized by indexing the ring pattern in the FFT.

After characterizing the crystallinity of $\mathrm{MoS}_{2}$, the cross-

A

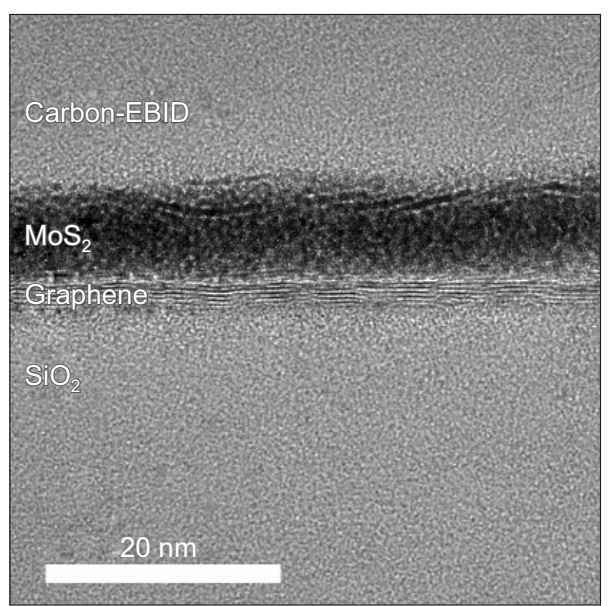

B

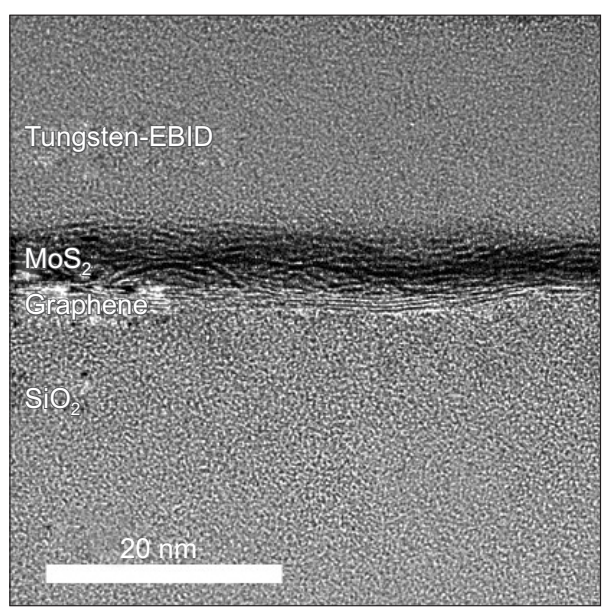

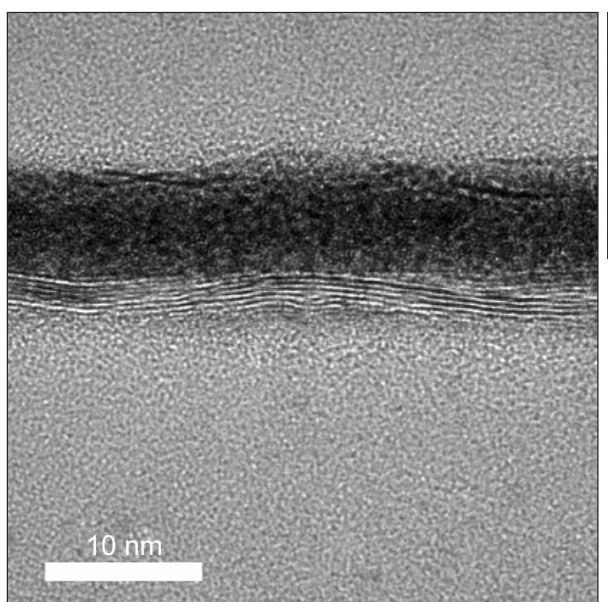
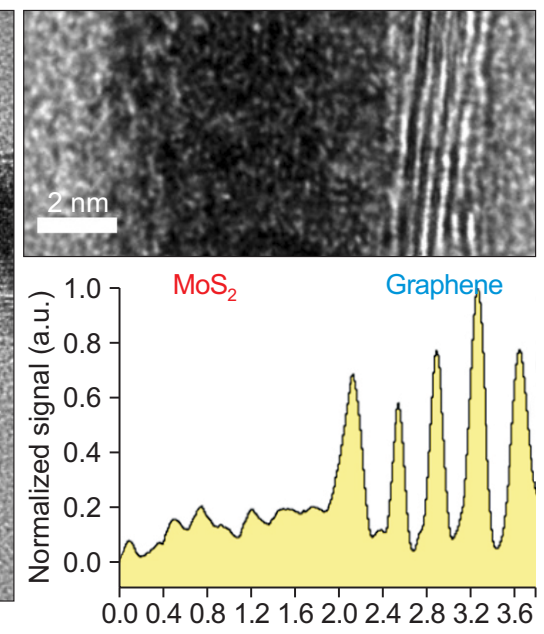

Distance $(\mathrm{nm})$

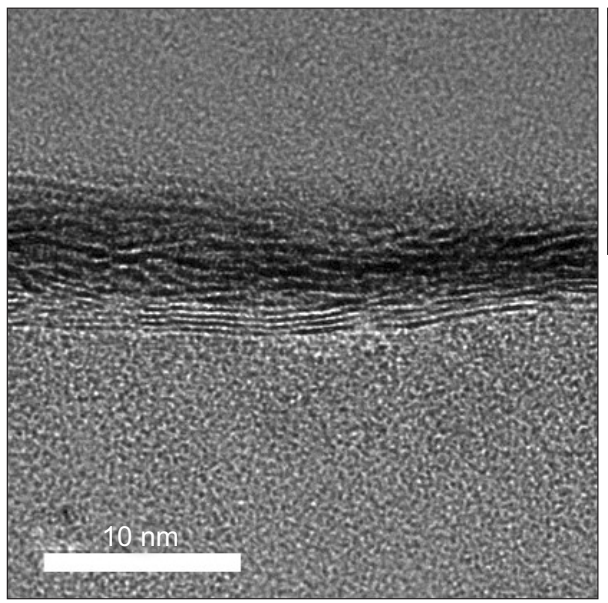

Fig. 3. The cross-sectional HR TEM image and line profile of $\mathrm{MoS}_{2}$-graphene heterostructure in TEM specimen fabricated using (A) carbon-EBID and (B) tungsten-EBID as protective layer. 
sectional TEM specimens fabricated by FIB were examined. Fig. 3 shows the cross-sectional HR TEM images of the specimens with carbon and tungsten protective layers. For the TEM specimen fabricated using carbon-EBID, the $\mathrm{MoS}_{2}$ layer was amorphized by the accelerated carbon source when the carbon layer was deposited using an electron beam, while graphene was observed without amorphization or damage, as shown in Fig. 3A. When measuring the d-spacing of each layer using the line profile of the HR TEM image, the d-spacing of the $\mathrm{MoS}_{2}$ layer could not be measured due to amorphization. The d-spacing of the graphene layer was approximately $0.35 \mathrm{~nm}$, which is consistent with that of typical graphite. For the TEM specimen fabricated using tungsten-EBID, the $\mathrm{MoS}_{2}$ and graphene layers were similar to their respective pristine states shown in Fig. 2 without amorphization or damage from the tungsten source (Fig. 3B). Unlike the large crystalline $\mathrm{MoS}_{2}$ synthesized by thermal CVD, discontinuous layers were observed because the $\mathrm{MoS}_{2}$ layer existed in the nanocrystalline state, as observed in the plan-view HR TEM image (Fig. 2). The d-spacing of the $\mathrm{MoS}_{2}$ and graphene layers measured by the line profile were $\sim 0.625$ and $\sim 0.35 \mathrm{~nm}$, respectively. The number of layers and crystallinity of the $\mathrm{MoS}_{2}$ were confirmed.

From the above results, the importance of selecting appropriate materials for the protective layer deposited by electron beam when fabricating TEM specimens using FIB is clear.

\section{SUMMARY}

In this study, we developed an optimized TEM specimen preparation method by generating an appropriate protective layer using EBID in the FIB method. By preparing successful TEM specimens using appropriate protective layers, it is possible to precisely analyze 2-D, biological, and organic materials which are especially vulnerable to damage by electron and ion beams.

\section{CONFLICT OF INTEREST}

No potential conflict of interest relevant to this article was reported.

\section{ACKNOWLEDGMENTS}

This study was supported by the Ministry of Trade, Industry \& Energy (MOTIE) [project number 10080625], the Korea Semiconductor Research Consortium (KSRC) support program for the development of future semiconductor devices, and NRF grants [NRF-2018R1A5A6075959] funded by the Korean government (MSIP). The authors are grateful for support from the SKKU Advanced Institute of Nanotechnology (SAINT) at Sungkyunkwan University.

\section{REFERENCES}

Ahn C, Lee J, Kim H U, Bark H, Jeon M, Ryu G H, Lee Z, Yeom G Y, Kim K, Jung J, Kim Y, Lee C, and Kim T (2015) Low-temperature synthesis of large-scale molybdenum disulfide thin films directly on a plastic substrate using plasma-enhanced chemical vapor deposition. Adv. Mater. 27, 5223-5229.

Cha H W, Kang M C, Shin K, and Yang C W (2016) Transmission electron microscopy specimen preparation of delicate materials using Tripod polisher. Appl. Microsc. 46, 110-115.

Garcia A, Raya A M, Mariscal M M, Esparza R, Herrera M, Molina S I, Scavello G, Galindo P L, Jose-Yacaman M, and Ponce A (2014) Analysis of electron beam damage of exfoliated MoS2 sheets and quantitative HAADF-STEM imaging. Ultramicroscopy 146, 33-38.

Giannuzzi L A and Stevie F A (1999) A review of focused ion beam milling techniques for TEM specimen preparation. Micron 30, 197-204.

John C B and Robert S (1984) The preparation of cross-section specimens for transmission electron microscopy. J. Electron Microsc.
Tech. 1, 53-61.

Kato N I (2004) Reducing focused ion beam damage to transmission electron microscopy samples. J. Electron Microsc. 53, 451-458.

Mkhoyan K A. Baston P E, Cha J, Schaff W J, and Silcox J (2006) Direct determination of local lattice polarity in crystals. Science 312, 1354, supplementary material.

Okuno H, Takeguchi M, Mitsuishi K, Guo X J, and Furuya K (2008) Sample preparation of GaN-based materials on a sapphire substrate for STEM analysis. J. Electron Microsc. 57, 1-5.

Stevie F A, Shane T C, Kahora P M, Hull R, Bahnck D, Kannan V C, and David $E$ (1995) Applications of focused ion beams in microelectronics production, design and development. Surf. Interface Anal. 23, 61-68.

Willians D B and Carter C B (2009) Transmission electron microscopy: A text book for materials science (Springer, New York). 